\title{
Fear of Falling and Associated Variables in Community-Dwelling Elderly
}

\author{
Akulwar Isha S. ${ }^{1}$, Dohadwala Sakina ${ }^{2}$ \\ ${ }^{1}$ Department of Neurosciences Physiotherapy, K. J. Somaiya College of Physiotherapy, Mumbai, India \\ ${ }^{2}$ Department of Physiotherapy, K. J. Somaiya College of Physiotherapy, Mumbai, India
}

Email address:

isha@somaiya.edu (Akulwar I. S.)

\section{To cite this article:}

Akulwar Isha S., Dohadwala Sakina. Fear of Falling and Associated Variables in Community-Dwelling Elderly. American Journal of Applied Psychology. Vol. 6, No. 6, 2017, pp. 153-157. doi: 10.11648/j.ajap.20170606.13

Received: June 10, 2017; Accepted: September 22, 2017; Published: November 2, 2017

\begin{abstract}
The purpose of this study was to determine the prevalence of fear of falling and its association with fall, avoidance of activity, balance deficits and risk of fall in community-dwelling older individuals. It was a cross-sectional study conducted in general community setting. Fifty community-dwelling elderly (mean age of $77.98 \pm 2.83$ years), ambulatory, without any severe medical conditions participated in the study. Main outcome measures of the study were fear of falling (FOF), fall and activity avoidance assessed through an interview-based questionnaire; balance assessed using Berg Balance Scale (BBS); balance confidence assessed using Activities-specific Balance Confidence (ABC) scale. FOF and associated avoidance of activity was reported by $60 \%$ and $52 \%$ of the elderly respectively. In subjects reporting FOF, $76 \%$ were fallers, $44 \%$ being non-fallers. BBS score of the subjects reporting FOF was significantly lower $(42.7 \pm 10.12)$ than the subjects without FOF $(53.65 \pm 3.51)$. Also, the subjects with FOF had a score below 46, the cut-off point for predicting risk of falling. In subjects having FOF, $56.66 \%$ had low risk of falls and $43.33 \%$ had medium risk of falls whereas all the subjects with no fear of fall had low risk of falls. Subjects FOF reported a significantly lower balance confidence on ABC scale as compared to subjects without FOF. Thus, it is concluded that FOF and associated avoidance of activity are highly prevalent in the community-dwelling older people. FOF is significantly associated with fall/s, balance deficits with an increased fall risk, avoidance of activity and low balance confidence in doing activities of daily living. FOF can be considered as a significant health problem of equal importance to a fall. This study highlights the importance of identifying FOF and addressing factors related to it in the rehabilitation of the elderly.
\end{abstract}

Keywords: Fear of Fall, Avoidance of Activities, Balance Confidence, Elderly

\section{Introduction}

Ageing is a progressive functional decline, or a gradual deterioration of physiological function with age [1]. According to population census 2011, India as the second most populous country in the world has approximately 104 million people at or over the age of 60 , constituting above $8.6 \%$ of total population. Falls are most commonly endured predicament by the elderly population in today's community and are an important cause of morbidity and mortality in the elderly. Many older persons experience psychological difficulties directly related to the fall [2]. Among these psychological consequences are fear of falling (FOF), loss of self-efficacy, activity avoidance and loss of self-confidence.
FOF can be considered as a significant health problem of equal importance to a fall and refers to the lack of selfconfidence that normal activities can be performed without falling [3]. FOF and associated avoidance of activity may lead to adverse consequences, like functional decline, restriction of social participation, decreased quality of life and increased risk of falling and institutionalization.

Both FOF and poor balance are established fall risk factors in the community-dwelling elderly. Studies suggest that older individuals who have a concern about their ability to avoid falling may have impaired balance. However, the relationship between FOF and balance impairments in elderly is not well established in the literature. Falls and FOF are potentially preventable and hence it is of importance to know the risk factors and consequences of FOF in elderly. Thus, the 
purpose of this study was to determine the prevalence of FOF and avoidance of activity and to investigate the association of this fear with fall, balance deficits and risk of fall in community-dwelling older individuals.

\section{Materials and Methods}

This was a cross-sectional study carried on a convenience sample of 50 community-dwelling elderly. Individuals above the age of 65 years, both males and females, ambulatory with or without an assistive device and living independently in the community, were recruited. Individuals were excluded from participation if they had (a) Symptomatic and complicated neurological, musculoskeletal, and cardiovascular diseases that accounts for imbalance; (b) Any recent surgical procedure for lower limbs; (c) Long term use of sedatives and hypnotics; (d) Any diagnosed psychiatric disorder; (e) Uncorrected visual or hearing impairments; (f) Vertigo or vestibular disorder; (g) Dementia. The procedures followed protocol and accord with the ethical standards of the institutional review board. Institutional review board approved design and conduct of the study and recruitment of research participants before the study was conducted. All the participants were volunteers and informed written and verbal consent was obtained from them before participation in the study. The compliance to the ethical principles and study protocol was monitored throughout the study by the primary investigator of the study.

Information regarding past history of fall, FOF, avoidance of activity and use of ambulatory aids was obtained through an interview-based questionnaire. In order to determine the prevalence, a single-item question assessed FOF (Are you afraid of falling?) and avoidance of activity (Do you avoid certain activities due to FOF?). A fall was defined as "an event which results in a person coming to rest inadvertently on the ground or other lower level and other than as a consequence of sustaining a violent blow; loss of consciousness; sudden onset of paralysis, as in a stroke; and an epileptic seizure" [4].

Berg Balance Scale (BBS) was used to assess balance and determine the risk of fall. This scale is a performance-based measure of balance in elderly and consists of 14 functional tasks [5]. Scoring uses a five point ordinal scale, with scoring ranging from 0 (unable) to 4 (safe and independent). Maximum score of the scale is 56 and according to the score 'risk of fall' is categorized as low (score 41-56), medium (score 21-40) and high (score 0-20).

Activities-specific Balance Confidence (ABC) scale was used to assess FOF and was administered through an interview. This 16-item scale includes questions addressing levels of balance confidence during performance of daily activities [6]. Subjects were asked to rate their level of confidence in doing the activity without losing balance or becoming unsteady on a scale of $0 \%$ to $100 \%$ where 0 stands for 'no confidence at all' and 100 stands for 'completely confident' to perform the specified activities. The total ABC Scale score is the average sum of the individual item scores and is expressed in percentage.

\section{Results and Discussion}

Descriptive statistics was performed on all subjects' demographic characteristics and variables regarding FOF, fall, balance and avoidance of activity (Table 1 and 2).

Table 1. Demographic characteristics of subjects.

\begin{tabular}{llll}
\hline & & Range & Mean \pm SD \\
\hline Age (in years) & & $75-85$ years & $77.98 \pm 2.832$ \\
Gender & & Number & $\%$ \\
& Male & 28 & 56 \\
Use of ambulatory aid & Female & 22 & 44 \\
\hline
\end{tabular}

Table 2. Characteristics of variables according to FOF status.

\begin{tabular}{llll}
\hline & & FOF & No \\
\cline { 3 - 4 } & & Yes & 70 \\
History of Fall (\% of subjects) & Yes & 76 & 30 \\
BBS score (mean \pm SD) & No & 44 & $53.65 \pm 3.51$ \\
Risk of fall & Low & $42.7 \pm 10.12$ & 100 \\
(\% of subjects) & Medium & 56.66 & - \\
ABC Score (mean \pm SD) & High & 43.33 & - \\
\hline
\end{tabular}

Variables distribution was tested using KolmogorovSmirnov test which exhibited significant departures from normality, and therefore nonparametric tests were used for all analyses (except for studying association between $\mathrm{ABC}$ and BBS score). Statistical significance was set at $\mathrm{P}<0.001$. Chisquare test was used to study univariate associations between fear of falling and history of fall. The results were significant
( $x=15.471, \mathrm{p}<0.0001)$ suggesting a relationship between fear of falling and history of fall. Mann Whitney U test was used to examine the relationship of FOF with BBS score and $\mathrm{ABC}$ scale score. The results were significant suggesting a relationship of FOF with ABC score $(p=0.001)$, and FOF with BBS score $(p=0.0041)$. The Pearson product moment coefficient of correlation was used to investigate the 
relationship between BBS score and $\mathrm{ABC}$ scale score. The results were significant $(\mathrm{p}<0.001$, for a one-tailed test, $\mathrm{r}=$ 0.81 ) indicating that there is a strong positive correlation between BBS score and ABC scale score.

The study aimed to determine the relationship of FOF with avoidance of activity, fall and balance deficits in communitydwelling elderly. In this sample of community-dwelling elderly, FOF was reported by $60 \%$ whereas activity avoidance due to FOF was reported by $52 \%$ of the subjects. Our finding is consistent with previous studies [7, 8, 9] reporting similar prevalence rates and that experiencing FOF to be strongly associated with avoidance of activity.

This sample included both fallers and non-fallers. History of fall was reported by $50 \%$ of the subjects. Experience of previous fall/s showed a significant association with FOF as a higher number of fallers than non-fallers reported of this fear. Having had at least one fall is reported in most studies as an independent risk factor for developing FOF [7, 10-12, 13-17]. In yet other studies using a prospective design, falling was reported as a consequence of FOF and FOF is considered to be predictive of falls. This increase in the risk of falling would be linked, according to some authors, to the activity restriction brought about by this fear [7, 14, 18, 19], a restriction that may cause, amongst other things, muscle atrophy [21], de-conditioning [14, 18, 19] and worse balance [19]. And, this in turn could feed the fear and avoidance [20]. This bi-directional relationship results in a harmful "spiraling risk of falls, FOF, and functional decline". Due to crosssectional design of the present study, casual relationship between fall and fear of fall could not be established.

For a long time, FOF was merely believed to be a result of the psychological trauma of a fall, also called 'post-fall syndrome' [2]. While fear of falling is mentioned frequently as an adverse outcome of falling, it is also commonly found among elderly persons, reported for example by between 12 and $65 \%$ of community-dwelling people, who have never sustained a fall. In the present study, $44 \%$ of the subjects reporting FOF had not yet experienced a fall. It is important to emphasize that, as mentioned in the literature, FOF and activity restriction is not limited to individuals with a history of fall. Etiology of FOF is multi factorial. Other psychological factors may be responsible for this fear in nonfallers.

One of the important objectives of this study was to investigate the relationship of balance deficits with FOF. It was found that the BBS score of the subjects reporting FOF was significantly lower $(42.7 \pm 10.12)$ than the subjects without FOF $(53.65 \pm 3.51)$. Also, the subjects with FOF had a BBS score below 45, the cut-off point for predicting risk of falling. Amongst the subjects who reported FOF, 56.66\% had low risk of fall and $43.33 \%$ had medium risk of fall. All the subjects without FOF had low risk of fall. Hence it is observed that FOF is significantly associated with poor balance abilities and increased risk of fall as assessed using BBS. This finding is consistent with past research that has demonstrated poorer performance of one-legged standing balance and anterior-posterior platform sway measures [21]; and poorer clinical balance scores as measured by the Performance-Oriented Mobility Assessment of Balance [22] by elderly with a fear of fall as compared with non-fearful subjects. Thus, balance impairments can be considered as a risk factor for development of FOF. However, due to crosssectional design of this study, this conclusion is subject to alternative interpretations of causal order. More recently William R. Young and A. Mark Williams reviewed the literature describing the fear of fall related alterations in the control of posture and gait and rationalized how FOF can influence attentional processes and jeopardize the acquisition and retention of sensory information necessary for the planning of safe stepping actions during complex locomotor tasks. [23]

In addition to direct measure of FOF, balance confidence which is a related construct to FOF was also assessed. Balance confidence is based on the broad psychology concept of self-efficacy and has specifically been defined as one's degree of belief in one's ability to avoid a loss of balance during activities of daily living [6]. We used ABC scale as it has been suggested as appropriate for active older adults because of its focus on high-level balancing and walking abilities $[6,24]$. Mean ABC scale score of all the subjects was $71 \%$ among which the subjects who had FOF had a significantly lower score $(63.17 \%)$ than the subjects who did not have FOF $(80.9 \%)$. Also, $52 \%$ of the subjects reported avoidance of activity due to FOF. This can be explained by the Bandura's self-efficacy theory [25, 26] which postulates that a person's perceived self-efficacy affects his or her activity performance. One's personal sense of ability and control influences one's motivation to engage in valued activities and occupations [27, 28]. Because selfefficacy theory directly links self-efficacy to participation in and performance of activity, addressing low balance confidence may indeed enhance activity engagement by elderly.

Although studies suggest that older individuals who have a concern about their ability to avoid falling may have impaired balance, our understanding of the relationship between balance confidence and actual balance ability is quite limited. To our knowledge, only two studies have attempted to explore this relationship. Myers and colleagues [24] investigated the association between balance confidence, as measured by the ABC Scale, and balance performance, as measured by static posturography, in elderly people. They reported a strong relationship between balance confidence and performance on medio-lateral sway, with subjects with higher balance confidence demonstrating less postural sway in standing than subjects with lower balance confidence. The use of static posturography as a measure of balance ability provides little information about a person's ability to maintain upright postural control while performing functional activities. In an effort to gain a better understanding of this phenomenon, we used BBS scale which assesses balance ability during functional tasks that challenge balance through multidirectional self-initiated perturbations, such as reaching, lifting, bending, and transfers. In accordance with findings of 
a previous study, a strong positive correlation of BBS score with $\mathrm{ABC}$ scale score is observed which proves the relationship between self-efficacy and actual balance ability. It also suggests that elderly people appear to have a good perception of problems with their own balance and may be making a rational assessment of their personal risk of sustaining falls and the associated consequences. An important aspect of this study is that both balance performance and balance confidence were assessed during functional tasks common to daily life.

In the present study, history of fall, balance deficits and low balance confidence are found to be significantly associated with FOF. Over time, this combination of factors may lead to further self-imposed limitations on physical activity. Activity restriction is, in itself, a risk factor for falls because it can lead to muscle atrophy, de-conditioning and poorer balance. Curtailment of activities can also lead to social isolation $[29,30]$. Thus, FOF can contribute to both functional decline and impaired quality of life. Interventions aimed at reducing FOF may help to reduce fall risk, reduce physical and social activity restriction, maintain independence and enhance quality of life in older adults, in particular elderly with balance problems. Multifactorial nature of FOF suggests that knowledge of these associated factors may be useful in developing multidimensional strategies to reduce FOF in elderly.

\subsection{Clinical Implications}

FOF is highly prevalent in community-dwelling elderly and thus, it should be specifically addressed in any rehabilitation program. It may be possible to decrease their FOF and self-imposed reduction in activity, which is hypothesized to be associated with further increases in frailty. Multiple factors have been identified to be associated with FOF in elderly. Results of this study indicate relationship of FOF with balance deficits and falls; both of which are potentially modifiable. To prevent or alleviate FOF and its consequences it is necessary to identify elderly at risk of developing FOF, fall and balance deficits. The association between FOF and balance deficits suggests that balance training should be an integral part of rehabilitation of the elderly, thus reducing FOF and further functional decline. Also, this implies that interventions aimed at improving balance deficits should not focus on balance alone but FOF as well. Thus, FOF can be used as an outcome measure to determine the effectiveness of balance training programs in elderly. Prevalence of FOF in non-fallers suggests that apart from intervening at the physical level, other psychological factors need to be addressed in fall prevention techniques. The findings of the current study are important for prevention purposes and may help health care professionals to identify people at risk for FOF and associated avoidance of activity. Healthcare professionals are vital links in the process of recognizing fearful and avoidant older people and referring them to interventions. We therefore recommend they inquire after FOF and associated avoidance of activity in patients at risk, for instance, elderly with balance deficits and history of fall. Subsequently, fearful and avoidant persons could be referred to interventions aimed at reducing FOF and increasing activity, such as a cognitive behavioral intervention, a taichi intervention or a home-based exercise intervention for community-living older people.

\subsection{Limitations}

Due to cross-sectional design of this study, no thorough conclusions can be made on causality regarding risk factors and consequences of FOF i.e. relationship between FOF on one hand; and fall and balance deficits on the other hand. Subjects were asked to report falls in the past, a retrospective recall which may be inaccurate [31], particularly in older adults. FOF can be a long lasting condition. Future studies should focus on prospective methods with adequacy of follow-up time to study the natural course of FOF. Apart from FOF, poor perceived general health and multiple falls are reported to be independently associated with avoidance of activity in the elderly. However, these factors were not considered in the present study. Also, psychological factors which can affect perception of FOF were not considered.

\section{Conclusion}

FOF and associated avoidance of activity are highly prevalent in the community-dwelling older people. FOF is significantly associated with fall/s, balance deficits with an increased fall risk, avoidance of activity and low balance confidence in doing activities of daily living. Remaining active is a critical component of successful aging and addressing FOF may be one avenue toward helping older adults maintain health and wellness. This study highlights the importance of identifying FOF in community-dwelling elderly and addressing the factors contributing to it in rehabilitation of elderly. There is a need for interventional studies to prevent and limit the consequences of FOF in elderly persons.

\section{Acknowledgements}

We would like to thank Mrs. Veena Krishnanand (Principal of K. J. Somaiya College of Physiotherapy, Mumbai) for her valuable support.

\section{Conflict of Interest Statement}

The authors confirm that there are no known conflicts of interest regarding the work described in the current manuscript.

\section{References}

[1] Partridge L, Mangel M. Messages from mortality: the evolution of death rates in the old. Trends Ecol Evol. 1999 Nov; 14(11):438-442.

[2] Legters K. Fear of falling. Phys Ther. 2002;82:264-72. 
[3] Tinetti ME, Richman D, Powell L. Falls efficacy as a measure of fear of falling. J Gerontol 1990; 45:239-43.

[4] Gibson, M. J., Andres, R. O., Isaacs, B., Radebaugh, T., WormPetersen, J. The prevention of falls in later life. A report of the Kellogg International Work Group on the prevention of falls by the elderly. Danish Medical Bulletin 1987;34 (Suppl 4): 1-24.

[5] Wood-Dauphinee S, Berg K, Bravo G, Williams JI. The Balance Scale: Responding to clinically meaningful changes. Canadian Journal of Rehabilitation 1997;10:35-50

[6] Powell LE, Myers AM. The Activities-specific Balance Confidence (ABC) Scale. J Gerontol Med Sci 1995;50(1): M28-34

[7] Friedman SM, Munoz B, West SK et al. Falls and fear of falling: Which comes first? A longitudinal prediction model suggests strategies for primary and secondary prevention. $J$ Am Geriatr Soc 2002;50:1329-35.

[8] Murphy SL, Dubin JA, Gill TM. The development of fear of falling among community-living older women: predisposing factors and subsequent fall events. J Gerontol A Biol Sci Med Sci 2003;58:M943-7.

[9] T. Hadjistavropoulos, K. Delbaere, T. D. Fitzgerald Reconceptualizing the role of fear of falling and balance confidence in fall risk J Aging Health, 23 (1) (2011), pp. 3-23

[10] Bosscher RJ, Raymakers ER, Trompe EA, et al. Angst om tevallen: psychometrische aspecten van Tinetti's falls efficacy scale. Tijdschr Gerontol Geriatr 2005;36:5-10.

[11] Hill KD, Schwarz JA, Kalogeropoulos AJ et al. Fear of falling revisited. Arch Phys Med Rehabil 1996;77:1025-9.

[12] Lachman ME, Howland J, Tennstedt $\mathrm{S}$ et al. Fear of falling and activity restriction: The survey of activities and fear of falling in the elderly (SAFE). J Gerontol B Psychol Sci Soc Sci 1998;53:43-50.

[13] Murphy SL, Dubin JA, Gill TM. The development of fear of falling among community-living older women: predisposing factors and subsequent fall events. J Gerontol A Biol Sci Med Sci 2003;58:M943-7.

[14] Howland J, Lachman ME, Peterson EW et al. Covariates of fear of falling and associated activity curtailment. Gerontologist 1998;38:549-55.

[15] Murphy SL, Williams CS, Gill TM. Characteristics associated with fear of falling and activity restriction in communityliving older persons. J Am Geriatr Soc 2002;50:516-20.

[16] Kressig RW, Wolf SL, Sattin RW et al. Associations of demographic, functional, and behavioral characteristics with activity-related fear of falling among older adults transitioning to frailty. J Am Geriatr Soc 2001;49:1456-62.

[17] Howland J, Peterson EW, Levin WC et al. Fear of falling among the community-dwelling elderly. $J$ Aging Health 1993;5:229-43.

[18] Hadjistavropoulos T, Martin RR, Sharpe D, Lints AC, McCreary DR, Asmundson GJ. A longitudinal investigation of fear of falling, fear of pain, and activity avoidance in community-dwelling older adults. $J$ Aging Health 2007;19:965-984.

[19] Gagnon N, Flint AJ, Naglie G, Devins GM. Affective correlates of fear of falling in elderly persons. Am J Geriatr Psychiatry 2005;13:7-14.

[20] Delbaere K, Crombez G, Vanderstraeten G, Willems T, Cambier D. Fear-related avoidance of activities, falls and physical frailty. A prospective community-based cohort study. Age Ageing 2004;33:368-373.

[21] Maki BE, Holliday PJ, Topper AK. Fear of Falling and postural performance in the elderly. J Gerontol A Biol Sci. Med Sci. 1991;46:M123-31.

[22] Tinetti ME. Performance-oriented assessment of mobility problems in elderly patients. $J$ Am Geriatr Soc.1986;34:119126.

[23] William R. Young, A. Mark Williams. How fear of falling can increase fall-risk in older adults: Applying psychological theory to practical observations. Gait \& Posture. 2015,41(1):7-12.

[24] Myers AM, Powell LE, Maki BE, Holliday PJ, Brawley LR, Sherk W. Psychological indicators of balance confidence: relationship to actual and perceived abilities. J. Gerontol. A: Biol. Sci. Med. Sci. 1996;51:M37-M43.

[25] Bandura A. Social foundations of thought and action: A social cognitive theory. Englewood Cliffs, NJ: Prentice Hall; 1986.

[26] Bandura A. Self-efficacy: The exercise of control. New York: W. H. Freeman; 1997.

[27] Kielhofner G. A model of human occupation: Theory and application. 4. Philadelphia: Lippincott Williams \& Wilkins; 2008.

[28] Peterson E, Kielhofner G, Tham K, von Koch L. Falls selfefficacy among adults with MS: A phenomenological study. OTJR: Occupation, Participation and Health 2010;30:148157

[29] Howland J, Lachman ME, Peterson EW, et al. Covariates of fear of falling and associated activity curtailment. Gerontologist 1998;38:549-55.

[30] Fletcher PC, Hirdes JP. Restriction in activity associated with fear of falling among community-based seniors using home care services. Age Ageing 2004;33(3):273-9.

[31] Peel N. Validating recall of falls by older people. Accident; Analysis and Prevention 2000; 32:371-372. 